\title{
On the station keeping of a Solar sail in the Elliptic Sun-Earth system
}

\author{
Ariadna Farrés and Àngel Jorba \\ Departament de Matemàtica Aplicada i Anàlisi \\ Universitat de Barcelona \\ Gran Via 585, 08007 Barcelona, Spain \\ E-mails: ari@maia.ub.es, angel@maia.ub.es
}

November 16, 2010

\begin{abstract}
In this work we focus on the dynamics of a Solar sail in the SunEarth Elliptic Restricted Three-Body Problem with Solar radiation pressure. The considered situation is the motion of a sail close to the $L_{1}$ point, but displacing the equilibrium point with the sail so that it is possible to have continuous communication with the Earth. In previous works we derived a station keeping strategy for this situation but using the Circular RTBP as a model.

In this paper we discuss the effect of the eccentricity in the region close to the sail-displaced $L_{1}$ point of the Circular RTBP. Then we show how to use the information on this dynamics to design a station keeping strategy. Finally, we apply these results to the GeoStorm mission, including errors in the sail orientation and on the estimation of the position of the sail in the simulations.
\end{abstract}

\section{Introduction}

One of the advantages of solar sails is that they open a wide new range of possible mission applications that cannot be achieved by a traditional spacecraft. For instance, we can place a solar sail high above the ecliptic plane, with the sail oriented such that the solar radiation pressure counteracts the gravitational attraction of the Earth (or any other planet). Thus, a sail allows to have a satellite hovering a pole of the planet. This mission concept 
was first introduced by Robert L. Forward in 1990 [7], who called it "Statite" (a spacecraft that does not orbit). Nowadays these ideas are being consider in the proposed Polar Observer and PolarSitter missions. These mission concepts would enable to have constant monitoring of the Polar regions of the Earth for climatological studies.

Moreover, if we consider the Circular Restricted Three Body Problem (CRTBP) and add the solar radiation pressure, families of artificial equilibria that replace the classical Lagrange points $L_{1, \ldots, 5}$ appear. For instance, if we consider that the sail is perpendicular to the Sun-sail line, we find an equilibrium point in the Earth-Sun line closer to the Sun that the classical $L_{1}$. If we change slightly the sail orientation, the different forces acting in the system will cancel out in different positions in the phase space, having a 2D family of artificial equilibria parametrised by the sail orientation (for further details $[11,12])$.

These artificial equilibria are possible target positions for mission applications. For example, placing several solar sails around the displaced $L_{1}$ could act as a solar shed, that could be used to cool down the Earth helping with the global warming [15]. Another interesting example is the GeoStorm mission $[10,17]$, the idea is to place a solar sail on an equilibrium point closer to the Sun than the Lagrangian point $L_{1}$ and displaced about $5^{\circ}$ from the Earth-Sun line, enabling observations of the Sun's magnetic field having a constant communication with the Earth. This would enable to alert of Geomagnetic storms, doubling the actual alert time of the ACE spacecraft (that is now orbiting on a Halo orbit around $L_{1}$ ).

All these missions require to maintain a solar sail in a fixed location. Nevertheless, most of these equilibria are unstable, hence a station keeping strategy is needed to maintain a solar sail close to equilibria for a long time.

In previous works $[2,4]$ we used dynamical systems tools to develop a station keeping strategy for this situation in the Circular RTBP model. The key point there was to understand the dynamics of the system for a fixed sail orientation, and see how it varies with the orientation of the sail. Then, this information was used to derive a sequence of changes on the sail orientation that keeps the sail close to equilibria. We already tested these algorithms with the GeoStorm and Polar Observer missions [2, 4]. During the simulations we considered the CRTBP + solar radiation pressure as a model. We also included random errors on the position and velocity determination as well as on the sail orientation to test the robustness of these algorithms. There it was shown that the most relevant errors (the ones with more impact on the dynamics) are the errors in the sail orientation.

In this paper we want to focus on the station keeping of a solar sail close to an equilibrium point, taking into account the eccentricity of the orbit of 
the Earth. Hence, the model considered here is the Elliptic RTBP plus the solar radiation pressure that, for simplicity, we will refer as ERTBP. This is a first approach of a more ambitious project where we want to consider a more complex model, including the gravitational effect of the whole solar system.

The main effect of the eccentricity on the Circular RTBP can be summarised as follows. It is known that, under quite general conditions, if you perturb an autonomous ODE with a T-periodic time dependent forcing, the fixed points are replaced by periodic orbits (of period $\mathrm{T}$ ). These orbits tend to the fixed points of the autonomous system when the amplitude of the forcing goes to zero. This is what happens in this case. In the ERTBP there is no longer a $2 \mathrm{D}$ family of equilibria parametrised by the sail orientation, but families of periodic orbits that replace these equilibria. In the Circular RTBP, for each sail orientation there is an equilibrium point. So, in the ERTBP there is a periodic orbit nearby. As the points we are considering are unstable, these periodic orbits are also unstable. Hence, we need to derive a station keeping strategy to remain close to it.

The station keeping is based on an extension of the results for fixed points presented in $[2,3]$. The main idea is to use the Floquet modes of the periodic orbit to find a periodic reference system that simplifies the tracking of the relative position of the solar sail with respect to the nominal periodic orbit and its stable and unstable invariant manifolds. Then, we can see how small changes on the sail orientation affect to the trajectory of the sail so we can use this information to derive a station keeping strategy.

The results are tested with the GeoStorm mission. To this end, we have randomly selected 1000 initial conditions close to the nominal periodic orbit of the sail and we have simulated the control strategy for each case. Moreover, we have added some random errors in the determination of the position (and speed) of the sail and in the execution of the manoeuvre (i.e., in the orientation of the sail). The control strategy manages to keep the sail close to the nominal orbit in all the considered cases.

The paper is organised as follows: Section 2 introduces the model for the forces acting on the sail and explains the dynamical model for the motion of the sail. In Section 3 we describe the effects of the eccentricity of the Earth on the phase space of the RTBP plus sail. In Section 4 we will discuss how to extend the station keeping strategy introduced in $[2,3]$ to this case, where we need to remain close to a periodic orbit. Finally, in Section 5 we will test the performance of this strategy on the GeoStorm mission. 


\section{Dynamical Model}

In this study we have taken the Sun-Earth Elliptical Restricted Three Body Problem (ERTBP) model, adding the solar radiation pressure due to the Solar sail. This is the first step of a project that plans to study the dynamics of a sail taking into account the effect of the whole Solar System.

The formulation that we use for the ERTBP is different from the one seen in the literature by McInnes et al. in $[8,1]$. Here we use an inertial reference system centred on the centre of mass of the two primaries, hence the two primaries are not fixed throughout time. We have chosen this reference frame because then the extension to the whole Solar System comes naturally.

\subsection{Solar Sail Model}

The acceleration given by the sail depends on the orientation of the sail and its efficiency. In a first approach, one can consider that the force due to the radiation pressure is produced by the reflection of the photons emitted by the Sun on the surface of the sail [11]. For a more realistic model, one should include the force produced by the absorption of photons by the sail [13]. The force produced by reflection is directed along the normal direction to the surface of the sail, while the absorption is strictly in the opposite direction of the Sun. This means that the direction of the resultant force should be tilted from the normal direction to the surface of the sail.

In this paper we consider the simplest model for the sail, that is, a flat and perfectly reflecting sail. Hence, we only consider the force induced by the reflection of the photons. Therefore, the force due to the solar radiation pressure will be in the normal direction to the surface of the sail, proportional to the projected area and inversely proportional to the distance to the Sun. According to [11], it takes the form,

$$
\vec{F}_{\text {sail }}=\beta \frac{(1-\mu)}{r_{P S}^{2}}\left\langle\vec{r}_{s}, \vec{n}\right\rangle^{2} \vec{n},
$$

where the constant $\beta$ is the sail lightness number, that accounts for the efficiency of the sail.

The sail orientation is parametrised by two angles, $\alpha$ and $\delta$, that can be defined in many ways $[11,9,14]$. Here we define them as follows: (i) $\alpha$ is the angle between the projection of the Sun-sail line, $\vec{r}_{s}$, and the normal vector to the sail, $\vec{n}$, on the ecliptic plane; (ii) $\delta$ is the angle between the projection of the Sun-sail line, $\vec{r}_{s}$, and the normal vector to the sail, $\vec{n}$, on the $y=0$ plane (see Fig. 1). 


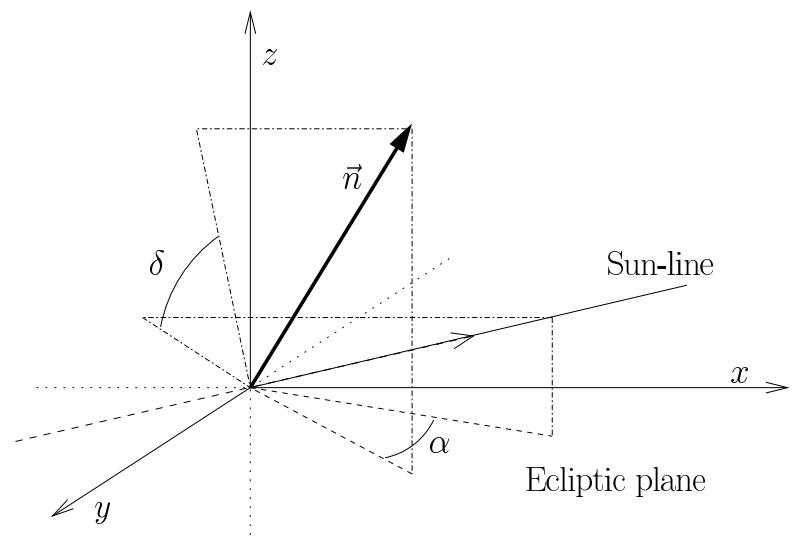

Figure 1: Graphic representation of the two angles $(\alpha, \delta)$ that define the sail orientation

We must mention that, when we study the dynamics of the system, we always consider that the sail orientation is fixed with respect to the Sun-sail line direction. In Section 3 we will describe some of the invariant objects that appear in the system for different fixed sail orientations.

\subsection{Restricted 3 Body Problem for a Solar Sail}

We assume that the solar sail is affected by the gravitational attraction of the two primaries (Earth and Sun) and the solar radiation pressure due to the sail. The motion of the two primaries is only affected by their mutual gravitational attraction, hence their motion can be described by the two-body problem.

A first approach for the Sail-Earth-Sun system is to consider that the two primaries follow a circular orbit around their mutual centre of mass. We will take a more realistic approach, considering the eccentricity in the motion of the two primaries, in the case of the Earth-Sun system we take $e=0.0167$.

Hence, we take an inertial reference frame with the origin at the centre of mass of the two primaries and with their orbit contained in the $Z=0$ plane. We also normalise the units of mass, distance and time so that the total mass of the system is 1, the semi-major axis of the Sun-Earth orbit is 
1 and the period of their orbit is $2 \pi$. The equations of motion are:

$$
\begin{aligned}
& \ddot{X}(t)=-(1-\mu) \frac{X(t)-X_{S}(t)}{r_{P S}^{3}}-\mu \frac{X(t)-X_{E}(t)}{r_{P E}^{3}}+\beta \frac{(1-\mu)}{r_{P S}^{3}}\left\langle\vec{r}_{s}, \vec{n}\right\rangle^{2} N_{X}, \\
& \ddot{Y}(t)=-(1-\mu) \frac{Y(t)-Y_{S}(t)}{r_{P S}^{3}}-\mu \frac{Y(t)-Y_{E}(t)}{r_{P E}^{3}}+\beta \frac{(1-\mu)}{r_{P S}^{3}}\left\langle\vec{r}_{s}, \vec{n}\right\rangle^{2} N_{Y}, \\
& \ddot{Z}(t)=-(1-\mu) \frac{Z(t)-Z_{S}(t)}{r_{P S}^{3}}-\mu \frac{Z(t)-Z_{S}(t)}{r_{P E}^{3}}+\beta \frac{(1-\mu)}{r_{P S}^{3}}\left\langle\vec{r}_{s}, \vec{n}\right\rangle^{2} N_{Z},
\end{aligned}
$$

where,

$$
\begin{aligned}
& r_{P S}=\sqrt{\left(X(t)-X_{S}(t)\right)^{2}+\left(Y(t)-Y_{S}(t)\right)^{2}+\left(Z(t)-Z_{S}(t)\right)^{2}}, \\
& r_{P E}=\sqrt{\left(X(t)-X_{E}(t)\right)^{2}+\left(Y(t)-Y_{E}(t)\right)^{2}+\left(Z(t)-Z_{S}(t)\right)^{2}},
\end{aligned}
$$

are the Sun - sail and Earth - sail mutual distances and $\vec{r}_{s}$ is the Sun sail direction. The normal direction to the surface of the sail is denoted by $\vec{n}=\left(N_{X}, N_{Y}, N_{Z}\right)$ and it depends on the two angles $\alpha$ and $\delta$ that define the sail orientation. The parameters $\mu$ and $\beta$ are the mass ratio of the system and the sail lightness number respectively.

The motion of the two primaries $\left(X_{S}(t), Y_{S}(t), Z_{S}(t)\right)$ and $\left(X_{E}(t), Y_{E}(t), Z_{E}(t)\right)$ is described by the two-body problem. We could take an analytical solution of the two-body problem but this would require to include Kepler's equation in the model. Hence, we have decided to include the equations of motion of the masses in the system:

$$
\begin{aligned}
& \ddot{X}_{S}=\mu \frac{X_{E}-X_{S}}{r_{S E}^{3}}, \quad \ddot{Y}_{S}=\mu \frac{Y_{E}-Y_{S}}{r_{S E}^{3}}, \quad \ddot{Z}_{S}=\mu \frac{Z_{E}-Z_{S}}{r_{S E}^{3}}, \\
& \ddot{X}_{E}=(1-\mu) \frac{X_{S}-X_{E}}{r_{S E}^{3}}, \quad \ddot{X}_{E}=(1-\mu) \frac{Y_{S}-Y_{E}}{r_{S E}^{3}}, \quad \ddot{X}_{E}=(1-\mu) \frac{Z_{S}-Z_{E}}{r_{S E}^{3}},
\end{aligned}
$$

where,

$$
r_{S E}=\sqrt{\left(X_{S}-X_{E}\right)^{2}+\left(Y_{S}-Y_{E}\right)^{2}+\left(Z_{S}-Z_{S}\right)^{2}},
$$

is the Sun-Earth distance. Taking suitable initial conditions we can force the desired motion for the two primaries.

The classical approach to the elliptical problem (without radiation pressure) can be found in Szebehely [16]. The usual reference frame is based on using a rotating-pulsating coordinate system, such that the primaries are kept in fixed positions on one of the axis. This is not our case: we use an inertial reference system so Sun and Earth are not fixed along time. 


\section{Equilibria of the System}

It is well known that the Circular RTBP, in synodical coordinates, has five equilibrium points, $L_{1, \ldots, 5}$. Three of them are on the line joining the two primaries and are called collinear points $\left(L_{1,2,3}\right)$. The other two form an equilateral triangle with the two primaries and are known as the triangular points $\left(L_{4,5}\right)$.

In this paper we consider two extra effects on this model. The first one is produced by the solar sail. As the orientation of the sail is assumed to be fixed in synodical coordinates (see Section 2.1), its effect does not depend on time, it only depends of the position of the sail and of its orientation. In this situation, the five equilibrium points still exist, but displaced from its previous position. That is, if we fix the sail lightness number $\beta$, there are 5 equilibrium points for each orientation $(\alpha, \delta)$ of the sail. Varying the two angles $(\alpha, \delta)$ we obtain a $2 \mathrm{D}$ surface of equilibria. If $\beta$ is small, this surface has five connected components, that merge for $\beta$ large enough. For more details, see $[11,12,2]$. In Fig. 2 we can see two slices of this surface for different values of $\beta$.
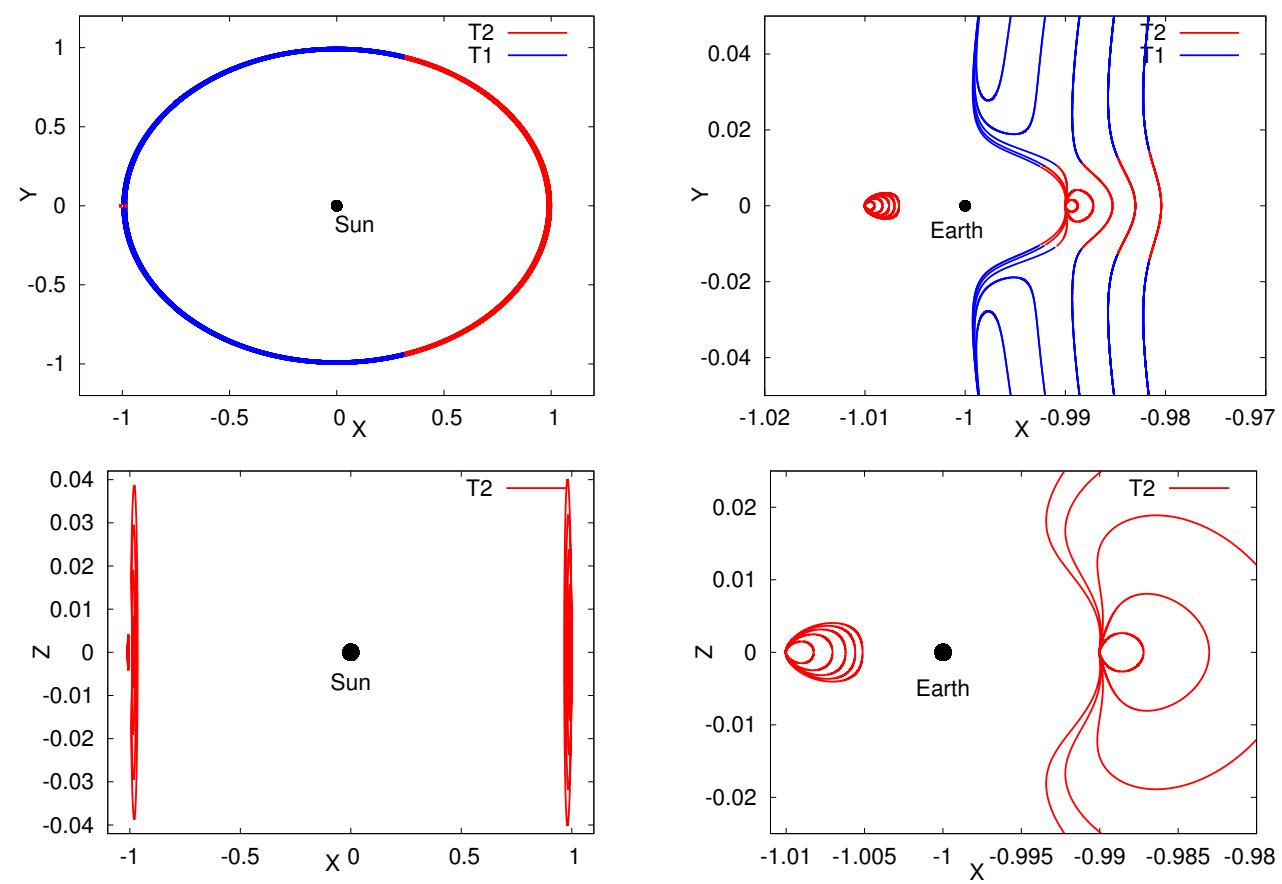

Figure 2: Slices of the family of equilibria for $\beta=0.001,0.005,0.01,0.05,0.1$, from left to right and top to bottom, slice $Z=0$, slice $Z=0$ zoom Earth region, slice $Y=0$ and slice $Y=0$ zoom Earth region. 
The second effect is due to the non-circular motion of the Earth. There are several possibilities to consider this effect. One is the elliptical problem (see, for instance, Szebehely [16]) where, using rotating and pulsating coordinates the two primaries are kept in a fixed position. Here we will look at the elliptical problem as a perturbation of the Circular RTBP. To explain the idea, let us consider the Circular RTBP in rotating coordinates such that the two primaries are fixed on the horizontal axis. If in the ERTBP we take the same rotation coordinate system, the gravitational attraction of the two masses (Sun and Earth) on the sail is the one of the Circular RTBP plus a small correction, because the two masses are not located in the same place as in the Circular RTBP, but slightly displaced due to their eccentricity. Hence, the ERTBP can be seen as a perturbation of the circular problem. Moreover, this perturbation depends on time in a periodic way, with the period of the motion of the masses. Hence, we have an autonomous problem, the Circular RTBP, plus a periodic time-dependent perturbation. In this case and under quite general conditions ${ }^{1}$, the fixed points of the autonomous problem are replaced by periodic orbits with the same period as the perturbation. We will place the sail on one of these periodic orbits.

We stress that we have done all the computations in inertial coordinates. In inertial coordinates, these periodic orbits are also periodic orbits with the same period as the masses. Hence, they can be computed by a standard multiple shooting method, using as a seed the circular orbit corresponding to the CRTBP plus sail.

In Fig. 3 we can see the different periodic orbits that for different values of the eccentricity replace the displaced $L_{1}$ point considered for the GeoStorm mission in an inertial (left) and in a rotating (right) reference system. We can see how the amplitude of these orbits increase as the eccentricity increases.

On the left hand side of Fig. 4 we see these periodic orbits for $\alpha \in$ $\left[0.274^{\circ}, 0.789^{\circ}\right]$ and $\delta=0^{\circ}$, and on the right hand side we can see the different periodic orbits for $\alpha=0^{\circ}$ and $\delta=\left[-2.86^{\circ}, 2.86^{\circ}\right]$. All of them for a fixed eccentricity $e=0.0167$.

\section{Station Keeping Strategy}

As it has been discussed in previous works by McInnes et. al [12, 10], the artificial equilibria that appear on the CRTBP when the solar radiation pressure is added are in an interesting location, for practical mission applications,

\footnotetext{
${ }^{1}$ Basically, a small enough perturbation and a non-resonance condition between the frequency of the elliptic motion of the masses with the frequencies of the linearised motion around the fixed point. These hypothesis are satisfied in the cases considered here.
} 

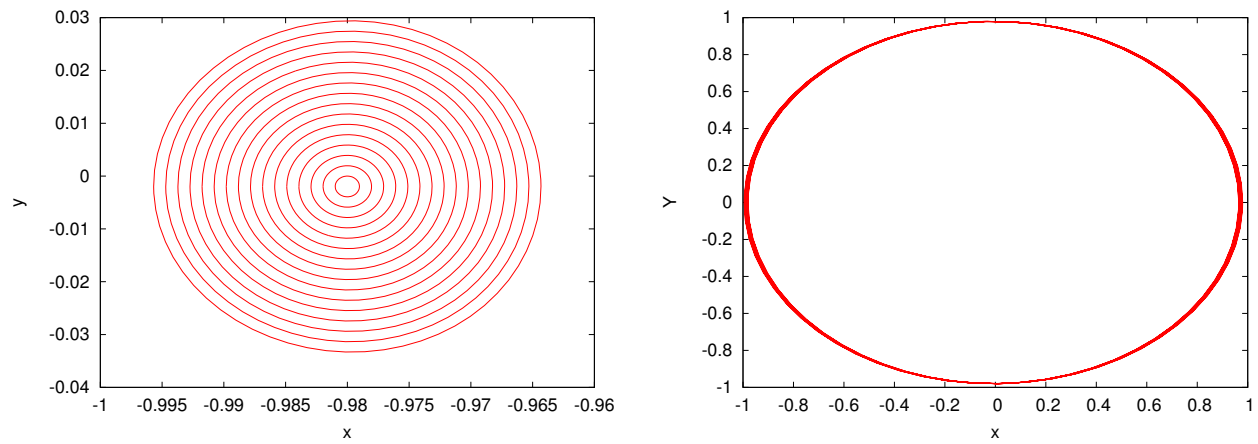

Figure 3: Continuation with respect to the eccentricity of the periodic orbits that replace the equilibrium point on the ERTBP for $e=0$ to $e=0.0167$. In a rotation reference system (left) and in an inertial reference system (right).

such as the GeoStorm or the Polar Observer. Nevertheless, they are unstable and a station keeping strategy must be used to maintain the sail close to equilibria.

In $[2,3,6]$, we already discussed how to derive station keeping strategies around unstable equilibria in the Circular RTBP using dynamical system tools. We also tested them and discussed their robustness when different sources of errors were included in the simulations (both on the position and velocity determination and the sail orientation).

As discussed in the previous section, when the eccentricity is included, there are no longer equilibrium points, they have been replaced by $2 \pi$ periodic orbits (with the standard units for the ERTBP, the period of the elliptic motion of Earth and Moon is $2 \pi$ ). We will place the sail close to one of these periodic orbits, as they have the same interesting properties regarding mission applications. Nevertheless, they are also unstable, hence we need a station keeping algorithm.

We recall that the key point of the strategies described in $[2,3]$ was to understand the geometry of the phase space and how it is affected by variations on the sail orientation. Hence, we will start by focusing on these periodic orbits and describing their linear dynamics and its variation for small variations on the sail orientation.

Before starting with the details, let us mention that a first extension of these strategies for periodic orbits is presented in [5], where we discuss the possibility of using these ideas to derive a control around a Halo-type orbit for a solar sail. The main difference with the work presented here is that for a fixed sail orientation there is a family of Halo-type orbits, while there is a unique orbit that replaces the fixed point for each eccentricity and sail 

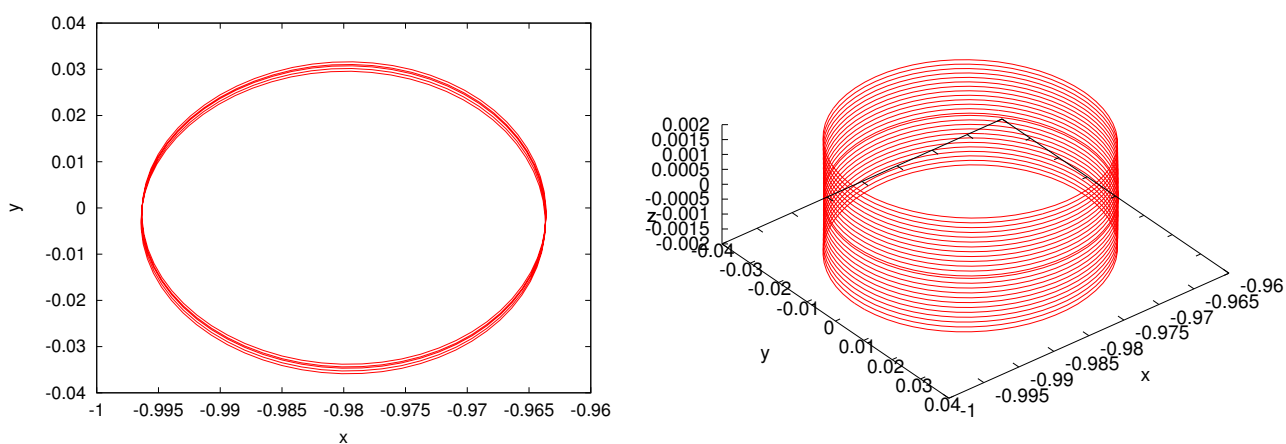

Figure 4: Continuation of the periodic orbits in the ERTBP with respect to the $\alpha$ and $\delta$ for a fixed eccentricity $e=0.0167$. All of them are plotted on an rotation reference system, on the left we have the continuation w.r.t. $\alpha$ and on the right the continuation w.r.t. $\delta$.

orientation. As we will see this simplifies the extension of the algorithm.

\subsection{Linear dynamics}

The first step to study the behaviour close to a periodic orbit is done throughout the first order variational equations. If we write the equations of motion in Eq. (2) as $\dot{y}=F(y, t)$, then the first variational equations are

$$
\dot{A}=D F(y(t)) A, \quad A \in \mathcal{L}\left(\mathbb{R}^{6}, \mathbb{R}^{6}\right),
$$

with the initial condition $A(0)=I d$.

We denote by $\phi$ the flow associated to Eq. (2) and $\phi_{\tau}\left(y_{0}\right)$ the image of the point $y_{0} \in \mathbb{R}^{6}$ after $\tau$ units of time. The solution $A(\tau)$ of Eq. (4) is the differential matrix, $D \phi_{\tau}\left(y_{0}\right)$, of $\phi_{\tau}\left(y_{0}\right)$ with respect to the initial condition $y_{0}$. For $h \in \mathbb{R}^{6}$ (with $\|h\|$ small enough), we have

$$
\phi_{\tau}\left(y_{0}+h\right)=\phi_{\tau}\left(y_{0}\right)+D \phi_{\tau}\left(y_{0}\right) \cdot h+O\left(|h|^{2}\right) .
$$

Therefore, $\phi_{\tau}\left(y_{0}\right)+A(\tau) \cdot h$ gives a good approximation of $\phi_{\tau}\left(y_{0}+h\right)$ provided that $h$ is small.

If we consider a $2 \pi$-periodic orbit, the variational matrix after one period, $A(2 \pi)$, is called the monodromy matrix associated to the orbit. The linear stability of the orbit is determined by the eigenvalues of this matrix.

For the periodic orbits that we consider in this study (see Section 3), the eigenvalues of the monodromy matrix $\left(\lambda_{1, \ldots, 6}\right)$ satisfy: $\lambda_{1,2}$ are real with $\lambda_{1}>1, \lambda_{2}<1$, the others $\lambda_{3,4,5,6}$ are complex pairs of conjugate eigenvalues, 
$\lambda_{3}=\bar{\lambda}_{4}$ and $\lambda_{5}=\bar{\lambda}_{6}$. As the system is volume preserving, $\prod_{i=0}^{6} \lambda_{i}=1$. We stress that although this system preserves volume (the divergence of the vector field is zero), it is not Hamiltonian.

These three pairs of eigenvalues have the following geometrical meaning:

- The first pair $\left(\lambda_{1}, \lambda_{2}\right)$ are related to the (strong) hyperbolic character of the orbit. The value $\lambda_{1}$ is the largest in absolute value, and is related to the eigenvalue $e_{1}(0)$, which gives the most expanding direction. After one period, a given distance to the nominal orbit in this direction is amplified in a factor of $\lambda_{1}$. Using $D \phi_{\tau}$ we can get the image of this vector under the variational flow: $e_{1}(\tau)=D \phi_{\tau} e_{1}(0)$. At each point of the orbit, the vector $e_{1}(\tau)$ together with the vector tangent to the orbit, span a plane that is tangent to the local unstable manifold $\left(W_{l o c}^{u}\right)$. In the same way $\lambda_{2}$ and its related eigenvector $e_{2}(0)$ are related to the stable manifold and $e_{2}(\tau)=D \phi_{\tau} e_{2}(0)$.

- The other two couples $\left(\lambda_{3}, \lambda_{4}=\bar{\lambda}_{3}\right)$ and $\left(\lambda_{5}, \lambda_{6}=\bar{\lambda}_{5}\right)$ are complex conjugate and their modulus is close to 1 (recall that Eq. (2) is a Hamiltonian system with a non-Hamiltonian perturbation). The monodromy matrix, restricted to the plane spanned by the real and imaginary parts of the eigenvectors associated to $\lambda_{3}, \lambda_{4}$ (and $\lambda_{5}, \lambda_{6}$ ) is a rotation with a small dissipation or expansion, so that the trajectories on these planes spiral inwards or outwards. The monodromy matrix restricted to these planes has the form

$$
\left(\begin{array}{rr}
\Delta_{i} \cos \Gamma_{i} & -\Delta_{i} \sin \Gamma_{i} \\
\Delta_{i} \sin \Gamma_{i} & \Delta_{i} \cos \Gamma_{i}
\end{array}\right)
$$

where $\Delta_{1,2}$ denotes the modulus of $\lambda_{3}$ and $\lambda_{5}$ respectively, and they are the rate of contraction or expansion for each period. $\Gamma_{1,2}$ denotes the argument of $\lambda_{3}$ and $\lambda_{5}$ respectively, and they account for the rotation around the periodic orbit (at each period).

- For the orbits considered here we have that $\left|\lambda_{3,4,5,6}\right| \ll\left|\lambda_{1}\right|$, hence the most expanding direction (by far) is given by $e_{1}(\tau)$.

To sum up, in a suitable basis the monodromy matrix associated to one of these periodic orbits can be written in the form,

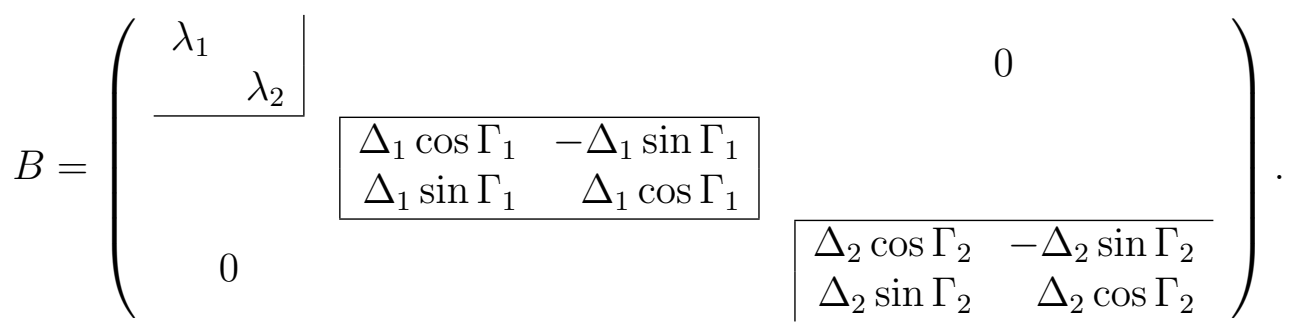


The functions $e_{i}(\tau)=D \phi_{\tau} \cdot e_{i}(0), i=1, \ldots, 6$, give an idea of the variation of the phase space properties in a small neighbourhood of the periodic orbit. We will use a modification of them called the Floquet modes $\bar{e}_{i}(\tau), i=$ $1, \ldots, 6$, six $2 \pi$-periodic functions that can easily be recovered by $e_{i}(\tau)$.

\subsubsection{The Floquet modes}

The Floquet modes provide a local reference system close to the periodic orbit, that is very useful to track the relative position between the spacecraft trajectory and the local unstable and stable invariant manifolds of the nominal orbit. The Floquet modes $\bar{e}_{i}(\tau)(i=1, \ldots, 6)$ are six periodic timedependent vectors (with the period of the orbit) such that, if we call $P(t)$ to the matrix that has the vectors $\bar{e}_{i}(\tau)$ as columns, then the change of variables $y=P(t) z$, takes the linearisation equation around the $2 \pi$-periodic orbit, $\dot{y}=A(\tau) y$, to an equation with constant coefficients $\dot{z}=B z$. Moreover, as they are periodic functions, they can be spanned as Fourier series and easily stored by their Fourier coefficients. Let us see how they are here.

We define the first and second Floquet mode taking into account that the rate of escape and approach, to the periodic orbit, along the unstable and stable manifolds is exponential:

$$
\begin{aligned}
& \bar{e}_{1}(\tau)=e_{1}(\tau) \exp \left(-\frac{\tau}{T} \ln \lambda_{1}\right), \\
& \bar{e}_{2}(\tau)=e_{2}(\tau) \exp \left(-\frac{\tau}{T} \ln \lambda_{2}\right) .
\end{aligned}
$$

The other pairs are computed taking into account that the monodromy matrix restricted to the plane generated by the real and imaginary parts of the eigenvectors associated to $\left(\lambda_{3}, \lambda_{4}\right)$ and $\left(\lambda_{5}, \lambda_{6}\right)$ is a rotation of angle $\Gamma_{1,2}$ and a dissipation/expansion by a factor of $\Delta_{1,2}$ :

$$
\begin{aligned}
& \bar{e}_{3}(\tau)=\left[\cos \left(-\Gamma_{1} \frac{\tau}{T}\right) e_{3}(\tau)-\sin \left(-\Gamma_{1} \frac{\tau}{T}\right) e_{4}(\tau)\right] \exp \left(-\frac{\tau}{T} \ln \Delta_{1}\right), \\
& \bar{e}_{4}(\tau)=\left[\sin \left(-\Gamma_{1} \frac{\tau}{T}\right) e_{3}(\tau)+\cos \left(-\Gamma_{1} \frac{\tau}{T}\right) e_{4}(\tau)\right] \exp \left(-\frac{\tau}{T} \ln \Delta_{1}\right), \\
& \bar{e}_{5}(\tau)=\left[\cos \left(-\Gamma_{2} \frac{\tau}{T}\right) e_{5}(\tau)-\sin \left(-\Gamma_{2} \frac{\tau}{T}\right) e_{6}(\tau)\right] \exp \left(-\frac{\tau}{T} \ln \Delta_{2}\right), \\
& \bar{e}_{6}(\tau)=\left[\sin \left(-\Gamma_{2} \frac{\tau}{T}\right) e_{5}(\tau)+\cos \left(-\Gamma_{2} \frac{\tau}{T}\right) e_{6}(\tau)\right] \exp \left(-\frac{\tau}{T} \ln \Delta_{2} \mid\right) .
\end{aligned}
$$

Where $T$ stands for the period of the periodic orbit, in this case, $T=2 \pi$.

In this new set of coordinates, the dynamics around the periodic orbit is quite simple. If $\phi_{\tau}\left(x_{0}\right)$ denotes the point on the periodic orbit at time $\tau$, then $\bar{e}_{1}(\tau)$ is the direction of the unstable manifold. When this base point follows 
the periodic orbit, the vector $\bar{e}_{1}(\tau)$ moves along the orbit following the (twodimensional) unstable manifold. In the same way, the vector $\bar{e}_{2}(\tau)$ follows the stable manifold along the orbit. For each point of the periodic orbit, the couple $\bar{e}_{3}(\tau), \bar{e}_{4}(\tau)$ spans a plane that is tangent to another invariant manifold of the orbit. This plane spans a three-dimensional manifold when the base point moves along the orbit. The dynamics on this manifold can be visualized as a spiral motion (towards the periodic orbit) on the plane $\left(\bar{e}_{3}(\tau), \bar{e}_{4}(\tau)\right)$ at the same time that the plane moves along the orbit. In a similar way, and $\bar{e}_{5}(\tau)$ and $\bar{e}_{6}(\tau)$ span another three-dimensional manifold, on which the dynamics is again a spiral motion (but now escaping from the periodic orbit) composed with the motion along the orbit. The growing (or compression) of these spiral motion is due to the real part of $\lambda_{3,4}$ and $\lambda_{5,6}$, which is nonzero but very small. For this reason the spiraling motion is very small (almost circular) and, to compute the control strategy, we will assume that this motion is not an spiral but a rotation. Of course, the simulations of the control strategy are done without this assumption, and the control is good enough to compensate the spiraling components (similar ideas were used in [2]).

\subsection{Variations of the sail orientation}

Now we know how the trajectories behave close to a periodic orbit for a fixed sail orientation. Let us then discuss how small changes on the sail orientation affect these trajectories.

In Section 3, we mentioned that small changes on the sail orientation imply no significant changes on the geometry of the phase space. When the sail orientation $(\alpha, \delta)$ is changed, the periodic orbits on the ERTBP still exist but their position is slightly displaced. Moreover, the qualitative behaviour around them is essentially the same for small changes in $\alpha$ and $\delta$.

We want to know how these orbits vary when we change the sail orientation. So we can use these information in our station keeping strategy. As we will deal with small variations on the sail orientation, a first order estimation of how these orbits vary is enough.

Let $P(x, \alpha, \delta)$ be a Poincaré section of the flow at time $2 \pi$ for a fixed sail orientation. The periodic orbits of the system are fixed points for these Poincaré section. As we know these fixed points will be parametrised by the sail orientation. If an orientation $(\alpha, \delta)$ is close to the actual orientation $(\alpha, \delta)$ we have that

$$
x(\alpha, \delta) \approx x\left(\alpha_{0}, \delta_{0}\right)+\frac{\partial x}{\partial \alpha} \cdot\left(\alpha-\alpha_{0}\right)+\frac{\partial x}{\partial \delta} \cdot\left(\delta-\delta_{0}\right),
$$


gives the (linear approximation to the) variation of the position of these fixed points in the Poincaré section. The derivatives $\partial x / \partial \alpha$ and $\partial x / \partial \delta$ can be found by solving $\left(D P_{\gamma}-I d\right) y=-P_{\gamma}$ with $\gamma=\alpha, \delta$ respectively.

Moreover, the vectors

$$
\begin{aligned}
& v_{\alpha}(\tau)=D \phi_{\tau}\left(x_{0}\right) \cdot \frac{\partial x}{\partial \alpha}\left(x_{0}\right)-\frac{\partial \phi_{\tau}}{\partial \alpha}\left(x_{0}\right), \\
& v_{\delta}(\tau)=D \phi_{\tau}\left(x_{0}\right) \cdot \frac{\partial x}{\partial \delta}\left(x_{0}\right)-\frac{\partial \phi_{\tau}}{\partial \delta}\left(x_{0}\right),
\end{aligned}
$$

are $2 \pi$ periodic functions that give us the linear variation of a periodic orbit $\phi_{\tau}\left(x_{0}\right)$ along time. Hence,

$$
\phi_{\tau}(x)=\phi_{\tau}\left(x_{0}\right)+v_{\alpha}(\tau)\left(\alpha-\alpha_{0}\right)+v_{\delta}(\tau)\left(\delta-\delta_{0}\right),
$$

is a good approximation for the new periodic orbits along time $(\tau)$ for small variations on $\alpha$ and $\delta$. These functions can be computed numerically, as they are periodic they can be spanned as Fourier series and easily stored by their Fourier coefficients.

\subsection{Control strategy}

Now let us assume that the solar sail is close to a given $2 \pi$-periodic orbit, $\phi_{\tau}\left(x_{0}\right)$, for a given fixed sail orientation $\alpha=\alpha_{0}, \delta=\delta_{0}$. The solar sail escapes along the unstable manifold $\left(W_{0}^{u}\right)$ and rotate along the other directions. When we change the sail orientation, $\alpha=\alpha_{1}, \delta=\delta_{1}$ the $2 \pi$-periodic orbit $\left(\phi_{\tau}\left(x_{1}\right)\right)$ is shifted. Now the trajectory will escape along the new unstable manifold $\left(W_{1}^{u}\right)$.

In order to maintain the solar sail trajectory close to the nominal periodic orbit $\left(\phi_{\tau}\left(x_{0}\right)\right)$ we need that the new unstable manifold $\left(W_{1}^{u}\right)$ takes the trajectory close to the stable manifold of $\phi_{\tau}\left(x_{0}\right)\left(W_{0}^{s}\right)$. If we can find a suitable sail orientation $\alpha_{1}, \delta_{1}$, then when we get close to $W_{0}^{s}$, we can restore the sail orientation to $\alpha_{0}, \delta_{0}$. The main idea is to repeat this process, having a sequence of changes on the sail orientation that manage to maintain the solar sails trajectory close to the desired periodic orbit. Nevertheless, one must take into account the rotating motion around the periodic orbit (see the section 4.1), because a sequence of changes on the sail orientation are a sequence of rotations around different periodic orbits on the centre projection, and this can result of an unbounded growth.

In order to find an appropriate new sail orientation $\left(\alpha_{1}, \delta_{1}\right)$ such that the trajectory comes close to the nominal periodic orbit $\phi_{\tau}\left(x_{0}\right)$ and the central component of the trajectory is kept bounded we follow the ideas described 
in $[2,4]$. There it is shown how to find the new sail orientation by solving a simple linear system. In this case we can apply similar ideas, the main difference is that the system to be solved depends on the instant of time when the manoeuvre has to be done. Let us see this in more detail.

Eq. (7) gives us the variation (for a fixed $\tau$ ) of the periodic orbits for small variations on the sail orientation. The Floquet modes $\bar{e}_{i}(\tau)$ for $i=1, \ldots, 6$ give us a periodic reference system useful to express the position of the solar sail with respect to the saddle and centre directions along time.

In Fig. 5 we see a schematic representation of the relative position of the new periodic orbit with respect to the sails trajectory in these reference system. We want the new periodic orbit to be placed on the appropriate side on the saddle projection w.r.t. the sail's trajectory (Fig. 5 left), and close to the periodic orbit on the centre projections (Fig. 5 right).
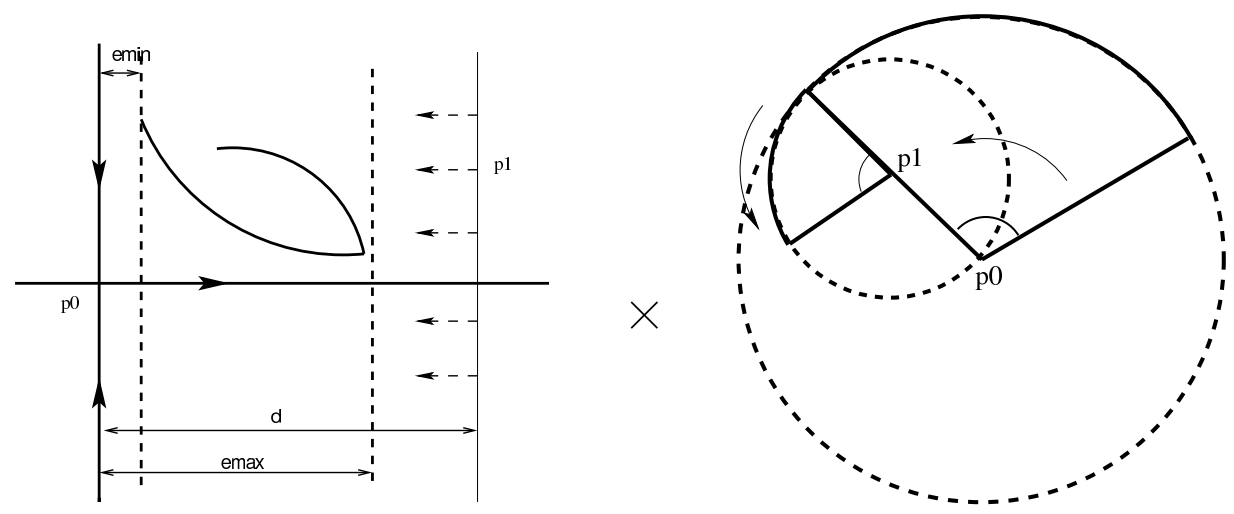

Figure 5: Ideal position for the new fixed point on the saddle (left) and centre (right) projections. Where $p_{0}$ represents the nominal periodic orbit $\left(\phi_{\tau}\left(x_{0}\right)\right)$ and $p_{1}$ the new periodic orbit $\left(\phi_{\tau}\left(x_{1}\right)\right)$ in the Floquet modes reference system.

The only inconvenient is that we are in a $6 \mathrm{D}$ phase space and the only have 2 parameters to play to find the new position for the periodic orbits. Hence, there might not be a periodic orbit where we want. We will chose the sail orientation that places the periodic orbit the closest to the desired position imposing some restrictions (for further details see $[2,4]$ ).

\subsection{Summary of the station keeping strategy}

Let us take a periodic orbit $\phi_{\tau}\left(x_{0}\right)$ for a fixed sail orientation $\alpha=\alpha_{0}, \delta=\delta_{0}$, and the Floquet modes associated to it $\bar{e}_{1}(\tau), \ldots, \bar{e}_{6}(\tau)$. This gives a $2 \pi$ periodic reference system to track along time the relative position of the trajectory with respect to the periodic orbit and the stable and unstable 
directions. During the control strategy we will always look at the trajectories in this reference system:

$$
\psi\left(t^{*}\right)=\phi_{t^{*}}\left(x_{0}\right)+\sum_{i=1}^{6} s_{i} \bar{e}_{i}\left(t^{*}\right),
$$

where $\left(s_{1}, \ldots, s_{6}\right)$ are the coordinates of the trajectory in this reference system. We also need to define the constants $\varepsilon_{\max }$ and $\varepsilon_{\min }$ that represent respectively, the maximum distance allowed to escape and "minimum" distance to the stable manifold of the periodic orbit on the saddle projection. By "minimum" we mean that the trajectory is close enough to the manifold to assume we have arrived there. These constants will depend on the mission objectives and the dynamical properties around the periodic orbit.

We start with the sail close to the nominal periodic orbit, $\phi_{\tau}\left(x_{0}\right)$, with $\alpha=\alpha_{0}, \delta=\delta_{0}$. Due to the instability the trajectory goes away along the unstable direction $\bar{e}_{i}(\tau)$. When $\left|s_{1}\right| \geq \varepsilon_{\max }$, we consider the sail to be too far from $\phi_{\tau}\left(x_{0}\right)$ so we set an appropriate new sail orientation $\alpha=\alpha_{1}, \delta=\delta_{1}$ that brings the probe back to a neighbourhood of $\phi_{\tau}\left(x_{0}\right)$. When $\left|s_{1}\right| \leq \varepsilon_{\text {min }}$, the sail is close to $W^{s}\left(x_{0}\right)$ and we change the sail orientation back to $\alpha=$ $\alpha_{0}, \delta=\delta_{0}$. This process is repeated on an on.

To find the appropriate new sail orientation $\alpha_{1}, \delta_{1}$ we just need to solve a linear system at each step. To have a better understanding on the conditions for these linear system you can look at $[2,3]$.

\section{Mission Application}

We have considered the GeoStorm Warning Mission [10, 17] as an example to test our strategies. The primary goal of the mission is to provide enhanced warning of geomagnetic storms to allow operation teams to take preventive actions to protect vulnerable systems. Currently predictions of future activity are made by the National Oceanic Atmospheric Administration (NOAA) Space Environment Centre in Colorado using terrestrial and real-time solar wind data obtained from the Advanced Compositions Explorer (ACE) spacecraft. The ACE spacecraft is stationed on a halo orbit near $L_{1}$, at about 0.01 $\mathrm{AU}$ from the Earth. From this position the spacecraft has continuous view of the Sun and communication with the Earth.

The enhanced storm warning provided by ACE is limited by the need to orbit the $L_{1}$ point and can only provide predictions of 1 hour in advance. However, since solar sails add an extra force to the dynamics of the orbit, the location of $L_{1}$ can be artificially displaced. The goal of GeoStorm is 
to station a solar sail twice as far from the Earth than $L_{1}$ while remaining close to the Earth-Sun line as can be seen in Fig. 6. Since the CME will be detected earlier than by ACE the warning times will be at least doubled.

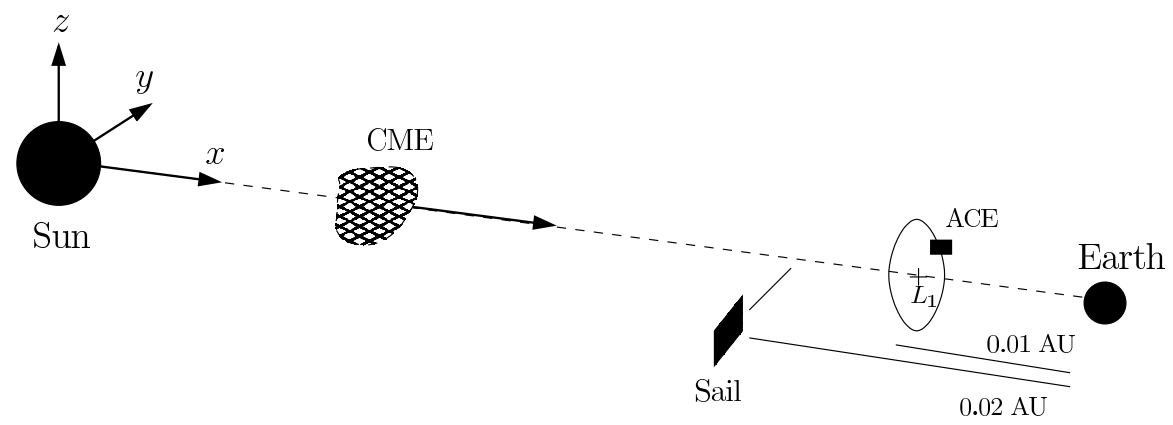

Figure 6: Schematic representation of the position of the GeoStorm Mission (not to scale).

If we want to displace a solar sail at a double distance from the classical Earth-Sun $L_{1}$ point, we need a sail lightness number $\beta=0.051689$ (which corresponds to $\left.a_{0} \approx 0.3 \mathrm{~mm} / \mathrm{s}^{2}\right)[11,17,10]$. Moreover, as we need constant communication with the Earth, its position must be displaced approximately $5^{\circ}$ from the Earth - Sun line. The equilibrium point that we find within this constraints in the Circular RTBP is unstable, hence a station keeping strategy is required.

In [2] we discussed the robustness of our strategy in the Circular RTBP for this mission, now we want to study its behaviour when we include the eccentricity in the dynamical model.

We have set the eccentricity of the Earth - Sun couple to $e=0.0167$ and computed the periodic orbit that replaces the fixed point that we had on the Circular RTBP. We will now use the strategy described in Section 4 to maintain the trajectory of the solar sail close to the periodic orbit that replaces the fixed point.

\subsection{Mission Results}

We have done a Monte Carlo simulation, taking 1000 initial conditions chosen in a random way close to the nominal periodic orbit. We have applied to each of them the control strategy up to 30 years. During the simulations we have measured the time between manoeuvre and the variation of the sail orientation $(\alpha, \delta)$ along time.

Taking the values of $\varepsilon_{\min }=5 . e-6$ and $\varepsilon_{\max }=5 . e-5(\mathrm{AU})$ we have that the average time between manoeuvres ranges between $\Delta t_{\text {max }}=151.03$ days 
and $\Delta t_{\min }=69.75$ days. The maximum average for the variation of the sail orientation is of 0.06 degrees for $\alpha$ and 0.003 degrees for $\delta$.

In Fig. 7 we can see the trajectory of a particular initial condition after applying the control strategy on the $X Y$-plane, the $X Z$-plane and the 3D trajectory. Notice that the trajectory is plotted in the rotational reference system of the Circular RTBP, and we can see how it remains close to the nominal periodic orbit along time.
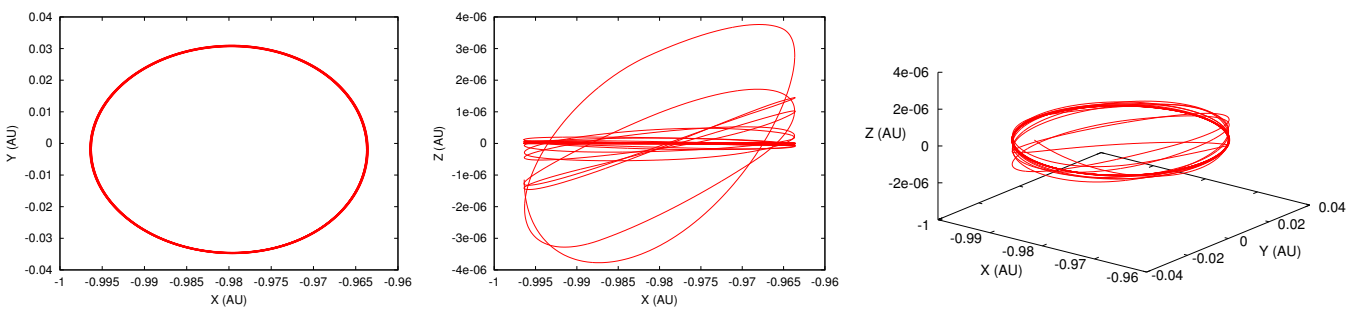

Figure 7: For the GeoStorm mission, in a rotational reference system. Trajectory followed by the sail for 30 years: $X Y$-projection (left), $X Z$-projection (middle) and $X Y Z$-projection (right).

In Fig. 8 we show the same trajectory as in Fig. 7 but on the Floquet mode reference system: the origin is at the periodic orbit $\phi_{x_{0}}(\tau)$, and the axis are given by $\left\{\bar{e}_{1}(\tau), \bar{e}_{2}(\tau), \bar{e}_{3}(\tau), \bar{e}_{4}(\tau), \bar{e}_{5}(\tau), \bar{e}_{6}(\tau)\right\}$. We can show the different projections: the saddle (generated by $\bar{e}_{1}(\tau), \bar{e}_{2}(\tau)$ ) (left) and the projection on the other two planes $\bar{e}_{3}(\tau), \bar{e}_{4}(\tau)$ (middle) and $\bar{e}_{5}(\tau), \bar{e}_{6}(\tau)$ (right). Notice how the trajectory on the saddle projection is a sequence of saddle connections between different periodic orbits, and that the other two projections are essentially a sequence of rotations that keep bounded along time.
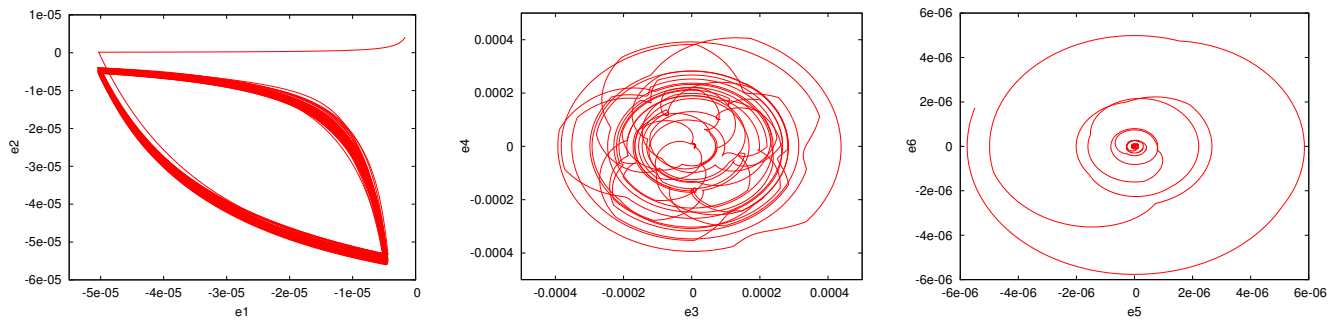

Figure 8: For the GeoStorm mission; In the Floquet's modes reference system. Trajectory followed by the probe for 30 years, saddle projection $\left.\left(\bar{e}_{1}(\tau), \bar{e}_{1} 2 \tau\right)\right)$ (left), centre projection generated by $\left(\bar{e}_{3}(\tau), \bar{e}_{4}(\tau)\right)$ and the other centre projection generated by $\left(\bar{e}_{5}(\tau), \bar{e}_{6}(\tau)\right)$. 
Finally in Fig. 9 we can see the variation of the two angles defining the sail orientation along time.
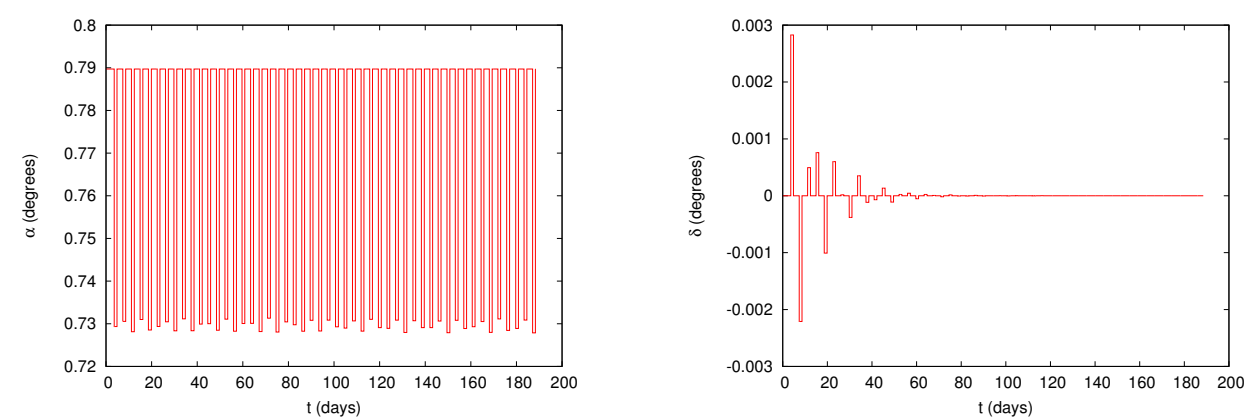

Figure 9: For the GeoStorm mission; Variation of the sail orientation along time: $\alpha$ (left) and $\delta$ (right).

\subsection{Error Sensitivity}

It is a known fact that during a mission the sail's position and velocity is not be determined exactly, and these errors may have an effect on the decisions taken by the control algorithm described in Section 4. Errors on the sail orientation are also made and have an important effect in the sail's trajectory. We want to see how robust our strategy is when we include all these effects in our simulations.

We will assume that all the errors follow a normal distribution with zero mean. We consider a precision on the position of the probe of $\approx 1 \mathrm{~m}$ in the space slant and $\approx 2-3$ milli-arc-seconds in the angle determination of the probe. The precision in speed is around $20-30$ microns/second. These errors are introduced every time the control algorithm asks for the position and velocity of the sail to decide if a manoeuvre should be done or not. As we will see the effect of these errors turns out to be almost negligible.

We also consider errors on the sail orientation, that are produced each time the sail orientation is changed. Hence, if the algorithm decides to change the sail orientation to $\alpha_{1}, \delta_{1}$, actually the new sail orientation turns to be $\alpha=\alpha_{1}+\epsilon_{\alpha}$ and $\delta=\delta_{1}+\epsilon_{\delta}$. Due to the lack of information on the literature regarding reasonable absolute values for $\epsilon_{\alpha}$ and $\epsilon_{\delta}$, we have performed several simulations considering different values for these errors, aiming to find the maximum error allowed for the sail orientation so that the trajectory is controllable.

We have performed a Monte Carlo simulations, using the same random initial conditions as before, but adding the uncertainty in the position, velocity 
and the sail orientation. The results are summarised in Table 1.

On Table 1 we show the results for the simulations when no errors are taken into account (line 1), when only errors on the position and velocity determination are considered (line 2) and when all the sources of error are taken into account (sail orientation and position + velocity determination). For each case we show the percentage of simulations that succeed (column 2), the average maximum and minimum time between manoeuvres (column 3), and the maximum and minimum variation on the sail orientation (columns 4 and 5).

Table 1: Statistics for 1000 simulations of the GeoStorm mission, with and without errors for the position determination and sail orientation. Type 1 errors only consider errors on the position and velocity determination and Type 2 errors also consider errors on the sail orientation, for different maximum bounds for these errors. Type $2 \mathrm{a}$ is $0.057^{\circ}=0.001 \mathrm{rad}$, Type $2 \mathrm{~b}$ is $0.286^{\circ}=0.005 \mathrm{rad}$ and Type $2 \mathrm{c}$ is $0.57^{\circ}=0.01 \mathrm{rad}$.

\begin{tabular}{lcccc}
\hline & $\%$ & $\Delta t$ & $\Delta_{\alpha}$ & $\Delta_{\delta}$ \\
\hline No Error & $100 \%$ & $69.75-151.03$ days & $0.058^{\circ}-0.0619^{\circ}$ & $0^{o}-0.003^{\circ}$ \\
Error Type 1 & $100 \%$ & $69.77-150.9$ days & $0.058^{\circ}-0.0619^{\circ}$ & $0^{o}-0.003^{\circ}$ \\
Error Type 2a & $100 \%$ & $67.28-166.9$ days & $0.057^{\circ}-0.063^{\circ}$ & $0^{o}-0.004^{o}$ \\
Error Type 2b & $100 \%$ & $58.05-349.7$ days & $0.049^{\circ}-0.069^{\circ}$ & $0.00009^{\circ}-0.018^{\circ}$ \\
Error Type 2c & $47.8 \%$ & $51.96-367.5$ days & $0.04^{\circ}-0.075^{\circ}$ & $0.0004^{o}-0.02^{\circ}$ \\
\hline
\end{tabular}

We can see that if we only consider errors on the position and velocity determination, the results are practically the same as when no sources of errors are considered on the simulations. The average time between manoeuvre and the maximum variations of the sail orientation are slightly different, but we have a $100 \%$ of success. This might not be the case when errors on the sail orientation are not taken into account.

As we already mentioned, as we do not have many information on reasonable values for the errors on the sail orientation, we have done several simulations considering different absolute magnitudes for the error on the sail orientation $\left|\varepsilon_{\alpha}\right|$ and $\left|\varepsilon_{\delta}\right|$. We have considered these values to be $0.001 \mathrm{rad}$, $0.005 \mathrm{rad}$ and $0.01 \mathrm{rad}$ (that correspond to $0.057^{\circ}, 0.286^{\circ}$ and $0.57^{\circ}$ ). The results are summarised in Table 1 in the lines corresponding to errors Type $2 \mathrm{a}, 2 \mathrm{~b}$ and $2 \mathrm{c}$ respectively. We can see that with errors of order 0.001rad and $0.005 \mathrm{rad}$ we always manage to maintain the solar sail close to the nominal orbit, this is not the case for larger errors.

Hence for this mission, the maximum error permitted for the sail orientation lies between $0.005 \mathrm{rad}$ and 0.01rad (i.e. $0.286^{\circ}$ and $0.57^{\circ}$ ). Further studies should be done to determine that maximum error. Moreover, possible 
changes on the station keeping algorithm could be done in order to improve these results. For instance, to use different conditions on the time when the changes on the sail orientation are made, or considering higher order approximations for the invariant objects and their variations w.r.t. changes on $\alpha$ and $\delta$.

Finally in Figs. 10 and 11 we can see the trajectory of the sail after applying the control strategy when all the different sources of errors are taken into account. In Fig. 10 we can see the trajectory on the $X Y$-plane, the $X Z$-plane and the $X Y Z$ projection, and in Fig. 11 we have the trajectory on the Floquet's modes reference system. And in Fig. 12 we can see the variation of the two angles defining the sail orientation along time.
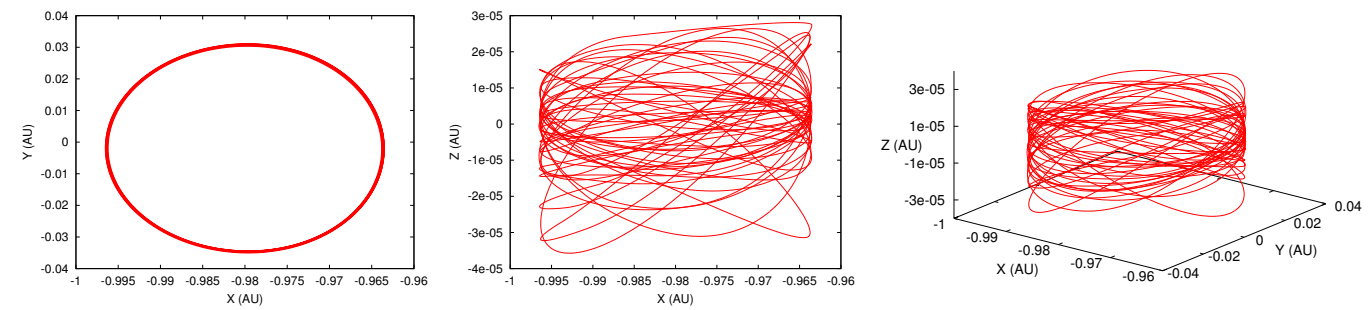

Figure 10: For the GeoStorm mission; In a rotational reference system and considering errors on the position + velocity determination and on the sail orientation. Trajectory followed by the sail for 30 years: $X Y$-projection (left), $X Z$-projection (middle) and $X Y Z$-projection (right).
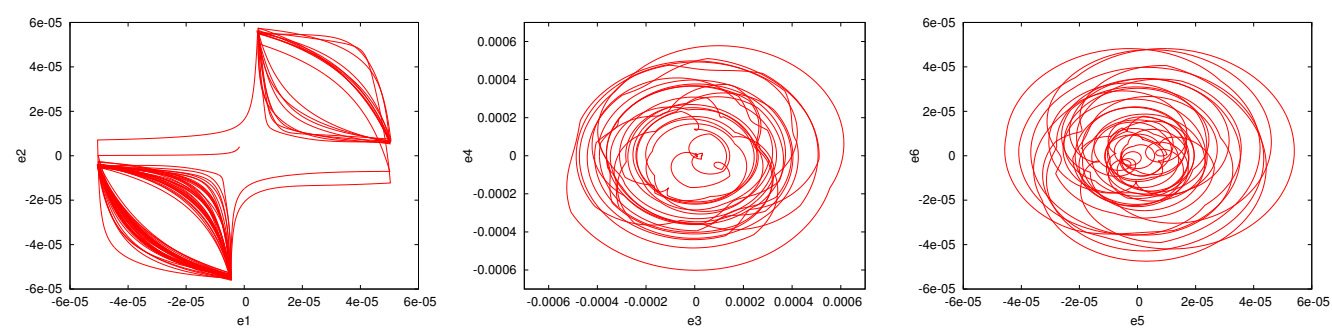

Figure 11: For the GeoStorm mission; In the Floquet's modes reference system and considering errors on the position + velocity determination and on the sail orientation. Trajectory followed by the probe for 30 years, saddle projection $\left(\bar{e}_{1}(\tau), \bar{e}_{1} 2 \tau\right)$ ) (left), centre projection generated by $\left(\bar{e}_{3}(\tau), \bar{e}_{4}(\tau)\right)$ and the other centre projection generated by $\left(\bar{e}_{5}(\tau), \bar{e}_{6}(\tau)\right)$. 

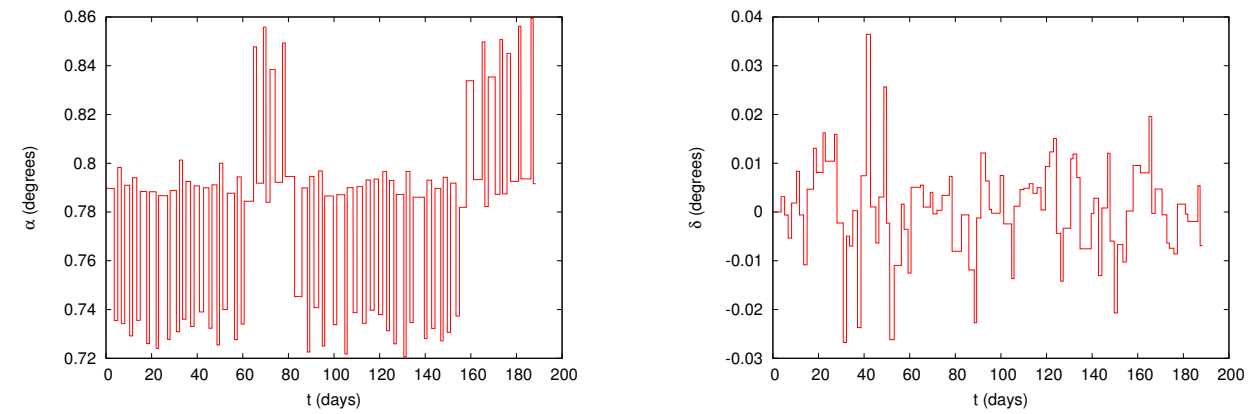

Figure 12: For the GeoStorm mission; Variation of the sail orientation along time: $\alpha$ (left) and $\delta$ (right).

\section{Conclusions}

In this work we have considered a solar sail near the $L_{1}$ point of the Earth-Sun system. As a concrete example, we have focused on the GeoStorm mission. The main novelty of the work is that, instead of using the Circular RTBP as a model, we have used the Elliptic RTBP model to account for the effect of the eccentricity of the Earth.

For this model we have computed a suitable periodic orbit for the GeoStorm mission, and we have studied the local dynamics around it. Then, we have used this information to derive a station keeping strategy. We have successfully tested this strategy including errors in the position determination and in the sail orientation.

The techniques used are not based on classical control theory but on dynamical systems tools. We already used these techniques in the Circular RTBP, and here we show how they extend naturally to more complex models like the Elliptic RTBP.

\section{Acknowledgments}

This work has been supported by the MEC grant MTM2009-09723 and the CIRIT grant 2009 SGR 67.

\section{References}

[1] Biggs, J.D. and McInnes, Colin Control of solar sail periodic orbits in the Elliptic Three-Body problem. Journal of Guidance, Control, and Dynamics , 32(1):318-320. 2009 
[2] A. Farrés and À. Jorba. Dynamical system approach for the station keeping of a solar sail. The Journal of Astronautical Science, 58(2), 199$230,2008$.

[3] A. Farrés and À. Jorba. Solar sail surfing along families of equilibrium points. Acta Astronautica, 63, 249-257, 2008.

[4] A. Farrés, Contribution to the Dynamics of a Solar Sail in the Earth-Sun System PHD Thesis, University of Barcelona, 2009.

[5] A. Farrés and À. Jorba. Dynamics of a solar sail near a Halo orbit. Acta Astronautica, 67:979-990, October-Novembre 2010.

[6] A. Farrés and À. Jorba. Sailing Between The Earth and Sun. In the proceedinds of the Second International Symposium on Solar Sailing, July 2010.

[7] R.L. Forward, Statite: A Spacecraft That Does Not Orbit. Journal of Spacecraft, 28(5), 606-611, 1990.

[8] Hexi, B. and McInnes, Colin Solar sail equilibria in the elliptical restricted three-body problem. Journal of Guidance, Control and Dynamics, 29(3), 538-543. 2006

[9] D.A. Lawrence and S. Piggott, Solar sailing trajectory control for sub-L1 stationkeeping. In AIAA-2004-5014, 2004.

[10] M. Macdonald and C. McInnes. A near - term road map for solar sailing. In 55th International Astronautical Congress, Vancouver, Canada, 2004.

[11] C. McInnes. Solar Sailing: Technology, Dynamics and Mission Applications. Springer-Praxis, 1999.

[12] C. McInnes, A. McDonald, J. Simmons, and E. MacDonald. Solar sail parking in restricted three-body system. Journal of Guidance, Control and Dynamics, 17(2), 399-406, 1994.

[13] A. Molostov and A. Shvartsburg. Heliocentric halos for a solar sail with absortion. Soviet Phys. Doklady, 37, 149-152, 1992.

[14] L. Rios-Reyes and D.J. Scheeres, Robust solar sail trajectory control for large pre-launch modelling errors. In 2005 AIAA Guidance, Navigation and Control Conference, August 2005. AIAA-2005-6173. 
[15] A. Roger, Feasibility of cooling the Earth with a cloud of small spacecraft near the inner Lagrange point $L_{1}$, Proceedings of the National Academy of Sciences, 103(46), 17184-17189, 2006.

[16] V. Szebehely. Theory of orbits. The restricted problem of three bodies. Academic Press, 1967.

[17] C.-W. L. Yen. Solar sail Geostorm Warning Mission design. In 14th AAS/AIAA Space Flight Mechanics Conference, Hawaii, February 2004. 